\title{
On the Monotonicity of the Isoperimetric Quotient for Parallel Bodies
}

\author{
Christian Richter ${ }^{1} \cdot$ Eugenia Saorín Gómez $^{2}$ (D)
}

Received: 25 May 2021 / Accepted: 6 October 2021 / Published online: 8 December 2021

(C) The Author(s) 2021

\begin{abstract}
The isoperimetric quotient of the whole family of inner and outer parallel bodies of a convex body is shown to be decreasing in the parameter of definition of parallel bodies, along with a characterization of those convex bodies for which that quotient happens to be constant on some interval within its domain. This is obtained relative to arbitrary gauge bodies, having the classical Euclidean setting as a particular case. Similar results are established for different families of Wulff shapes that are closely related to parallel bodies. These give rise to solutions of isoperimetric-type problems. Furthermore, new results on the monotonicity of quotients of other quermassintegrals different from surface area and volume, for the family of parallel bodies, are obtained.
\end{abstract}

Keywords Parallel body Tangential body $\cdot$ Volume $\cdot$ Surface area .

Quermassintegral · Isoperimetric quotient · Isoperimetric inequality · Gauge body ·

Wulff shape

Mathematics Subject Classification Primary 52A40; Secondary 52A20 - 52A21 . $52 \mathrm{~A} 38 \cdot 52 \mathrm{~A} 39 \cdot 52 \mathrm{~B} 60$

The second author is partially supported by MICINN/FEDER project PGC2018-097046-B-I00 and Fundación Séneca project 19901/GERM/15.

Eugenia Saorín Gómez

esaoring@uni-bremen.de

Christian Richter

christian.richter@uni-jena.de

1 Institute of Mathematics, Friedrich Schiller University, 07737 Jena, Germany

2 ALTA Institute for Algebra, Geometry, Topology and their Applications, University of Bremen, 28334 Bremen, Germany 


\section{Introduction}

Inner parallel bodies of convex bodies have been the object of recent studies with different flavors [7,12-15,18,20]. Classical references on inner parallel bodies (e.g. $[2,5,6,10,11,25])$ along with their role in the proofs of fundamental results in the theory of convex bodies make inner parallel bodies an essential object within classical convex geometry [26, Section 7.5] and beyond (see e.g. [8,17,23]). Inner parallel bodies and their properties were thoroughly studied by Bol [2], Dinghas [5] (see also [10,11]) and later by Sangwine-Yager [25].

Let $\mathcal{K}^{n}$ denote the family of convex bodies in $\mathbb{R}^{n}$, i.e. of nonempty compact convex subsets of the Euclidean space $\mathbb{R}^{n}$, and let $\mathcal{K}_{n}^{n}$ be its subfamily of convex bodies with nonempty interior. Let $B_{n}$ be the $n$-dimensional unit ball and $\mathbb{S}^{n-1}$ the corresponding unit sphere. The volume of a convex body $K \subseteq \mathbb{R}^{n}$, i.e. its $n$-dimensional Lebesgue measure, is denoted by $\operatorname{vol}(K)$. The measure of its boundary, i.e. its surface area or $(n-1)$-dimensional Hausdorff measure, is denoted by $\mathrm{S}(K)$.

For $K \in \mathcal{K}_{n}^{n}$, the classical isoperimetric quotient is the ratio

$$
\mathrm{I}(K)=\frac{\mathrm{S}(K)^{n}}{\operatorname{vol}(K)^{n-1}} .
$$

Let $K, E \in \mathcal{K}^{n}$. The inradius $\mathrm{r}(K ; E)$ of $K$ relative to $E$ is the largest possible factor of a homothety mapping $E$ into $K$, i.e.

$$
\mathrm{r}(K ; E)=\sup \left\{r \geq 0: \text { there is } x \in \mathbb{R}^{n} \text { with } x+r E \subseteq K\right\} .
$$

For $-\mathrm{r}(K ; E) \leq \lambda \leq 0$, the inner parallel body $K_{\lambda}$ of $K$ relative to $E$ at distance $|\lambda|$ is the Minkowski difference of $K$ and $|\lambda| E$, i.e.

$$
K_{\lambda}=K \sim|\lambda| E=\left\{x \in \mathbb{R}^{n}:|\lambda| E+x \subseteq K\right\} \in \mathcal{K}^{n} .
$$

If $E=B_{n}$, then $K_{-\mathrm{r}\left(K ; B_{n}\right)}$ is the set of incentres of $K$. For any $E \in \mathcal{K}^{n}$, the set $K_{-\mathrm{r}(K ; E)}$ has dimension strictly less than $n$ (see [3, p. 59]). The inner parallel sets complement the family of outer parallel sets $K_{\lambda}, \lambda \geq 0$, relative to $E$ at distance $\lambda$, that are defined as the Minkowski sums $K_{\lambda}=K+\lambda E$.

We prove that $\mathrm{I}(K) \geq \mathrm{I}\left(K_{\lambda}\right)$ for $-\mathrm{r}(K ; E)<\lambda \leq 0$ by investigating the behaviour of the isoperimetric quotient as a function of the real parameter $\lambda$. Our main result in this direction is the following (see Corollary 3.5), extending the well-known result for outer parallel bodies [16, Remark 4.4] to inner ones.

Theorem 1.1 Let $K \in \mathcal{K}_{n}^{n}$. Then the isoperimetric quotient $\mathrm{I}\left(K_{\lambda}\right)$ is monotonically decreasing for $\lambda \in\left(-\mathrm{r}\left(K ; B_{n}\right), \infty\right)$.

We will also analyse the situation where the function $\lambda \mapsto \mathrm{I}\left(K_{\lambda}\right)$ is constant in some subinterval of $\left(-\mathrm{r}\left(K ; B_{n}\right), \infty\right)$, characterizing the convex bodies $K \in \mathcal{K}_{n}^{n}$ for which this behaviour occurs.

We notice that the surface area is implicitly defined with respect, or relative, to the Euclidean unit ball, see Section 2 for details. Indeed, the two magnitudes, volume 
and surface area, involved in the isoperimetric quotient are particular cases of the socalled quermassintegrals of a convex body, and can be defined relative to a fixed body $E \in \mathcal{K}_{n}^{n}$, for which we refer the reader to Section 2 and the references therein.

In this note, for a fixed reference body $E \in \mathcal{K}_{n}^{n}$ - usually called gauge, we investigate the behaviour of the relative isoperimetric quotient function $\frac{\mathrm{S}\left(K_{\lambda} ; E\right)^{n}}{\operatorname{vol}\left(K_{\lambda}\right)^{n-1}}$ (see Section 2 for details) of the family of parallel bodies of the convex body $K \in \mathcal{K}_{n}^{n}$ with respect to $E$. In [16] (cf. [7]) the authors prove that, under certain boundary restrictions of the involved convex bodies or under certain differentiability conditions of the involved magnitudes, respectively, the relative isoperimetric quotient $\frac{\mathrm{S}\left(K_{\lambda} ; E\right)^{n}}{\operatorname{vol}\left(K_{\lambda}\right)^{n-1}}$ is monotonically decreasing for $\lambda \in(-\mathrm{r}(K ; E), \infty)$. For $\lambda \geq 0$, i.e. for outer parallel bodies, this is a direct consequence of the relative Steiner formula (2.2) [16, Remark 4.4]. We point out that in the planar case $K, E \in \mathcal{K}_{2}^{2}$ the monotonicity in the whole domain $(-\mathrm{r}(K ; E), \infty)$ seems to be treated as folklore, and to the best of our knowledge, there is no precise reference containing this result.

Here, we prove that no assumption on the convex bodies $K, E \in \mathcal{K}_{n}^{n}$ is necessary to have monotonicity on the total domain $(-\mathrm{r}(K ; E), \infty)$. Moreover, we characterize all convex bodies for which the quotient is constant in some interval of its domain (see Theorem 3.4).

In the above result, the family $\left(K_{\lambda}\right)_{-\mathrm{r}(K ; E) \leq \lambda \leq 0}$ of inner parallel bodies is extended by the family $\left(K_{\lambda}\right)_{\lambda \geq 0}$ of outer parallel bodies. In Section 4 , we introduce other natural extensions of $\left(K_{\lambda}\right)_{-\mathrm{r}(K ; E) \leq \lambda \leq 0}$ to parameters $\lambda \geq 0$, based on particular Wulff shapes. Also the isoperimetric quotients of these families are monotonically decreasing (see Theorem 4.5). This allows us to characterize isoperimetrically optimal bodies $K$ with restricted outer normals, again relative to a gauge body $E$ (see Section 4).

Finally, we investigate the behaviour of further quotients of magnitudes for parallel bodies intimately connected to the isoperimetric quotient, both for the classical and relative cases (see Section 5). This continues work of [16].

We would like to point out that in the meantime Graziano Crasta published a paper in arXiv.org which contains our central result of Theorem 3.4 [4, Theorem 1.2]. According to the arXiv.org web, his paper was uploaded in January 2021. The actual version of the present paper, up to minor typos, coincides with the version we uploaded to arXiv.org by March 2020.

\section{Background}

We write $K_{\lambda}$ to denote the inner and outer parallel bodies of $K \in \mathcal{K}^{n}$ relative to the gauge body $E \in \mathcal{K}_{n}^{n}$,

$$
K_{\lambda}= \begin{cases}K \sim|\lambda| E & \text { for }-\mathrm{r}(K ; E) \leq \lambda \leq 0 \\ K+\lambda E & \text { for } 0 \leq \lambda<\infty\end{cases}
$$

For $K \in \mathcal{K}^{n}$ and $u \in \mathbb{R}^{n}, h_{K}(u)=\sup \{\langle x, u\rangle: x \in K\}$ denotes the support function of $K \in \mathcal{K}^{n}$, where $\langle\cdot, \cdot\rangle$ stands for the standard Euclidean scalar product in $\mathbb{R}^{n}$ (see e.g. [26, Section 1.7]). For $u \in \mathbb{R}^{n} \backslash\{0\}$ and $\alpha \in \mathbb{R}$, we define the halfspace 
$H_{u, \alpha}^{-}=\{x \in \mathbb{R}:\langle x, u\rangle \leq \alpha\}[26, \mathrm{p} . \mathrm{xx}]$. Then the above parallel bodies $K_{\lambda}$ can be equivalently defined as

$$
K_{\lambda}=\bigcap_{u \in \mathbb{S}^{n-1}} H_{u, h_{K}(u)+\lambda h_{E}(u)}^{-}
$$

for all $\lambda \in[-\mathrm{r}(K ; E), \infty)$ [25, Lemma 4.1], [26, formula (3.19) and Theorem 1.7.5(a)].

The so-called relative Steiner formula states that the volume of the outer parallel body $K+\lambda E$ is a polynomial of degree $n$ in $\lambda \geq 0$,

$$
\operatorname{vol}(K+\lambda E)=\sum_{i=0}^{n}\left(\begin{array}{l}
n \\
i
\end{array}\right) \mathrm{W}_{i}(K ; E) \lambda^{i} .
$$

The coefficients $\mathrm{W}_{i}(K ; E)$ are called the relative quermassintegrals of $K$, and they are just a special case of the more general mixed volumes, for which we refer to [26, Section 5.1] and [9, Sections 6.2, 6.3] (where [26] uses the notation $V_{(i)}(K, E)$ for $\left.W_{i}(K ; E)\right)$. In particular, we have $\mathrm{W}_{0}(K ; E)=\operatorname{vol}(K)$ and $\mathrm{W}_{n}(K ; E)=\operatorname{vol}(E)$.

Applying (2.2) to both sides of $K+(\lambda+\mu) E=(K+\lambda E)+\mu E$ for $\lambda, \mu \geq 0$ and equating the coefficients of $\mu^{i}$ in both polynomials, we obtain the values of the relative $i$-th quermassintegrals of $K+\lambda E$, namely

$$
\mathrm{W}_{i}(K+\lambda E ; E)=\sum_{k=0}^{n-i}\left(\begin{array}{c}
n-i \\
k
\end{array}\right) \mathrm{W}_{i+k}(K ; E) \lambda^{k},
$$

for $\lambda \geq 0$ and $i=0, \ldots, n$ (cf. [9, Theorem 6.14]).

If $E=B_{n}$, the polynomial on the right-hand side of (2.2) becomes the classical Steiner polynomial, see [27]. Then the coefficient $\left(\begin{array}{l}n \\ 1\end{array}\right) \mathrm{W}_{1}\left(K ; B_{n}\right)$ in (2.2) happens to be the surface area $\mathrm{S}(K)$ if $K \in \mathcal{K}_{n}^{n}$. This motivates the definition of the surface area of $K$ relative to $E \in \mathcal{K}_{n}^{n}$ by

$$
\mathrm{S}(K ; E)=n \mathrm{~W}_{1}(K ; E)
$$

(see e.g. [11, Section 5.1.2]) and the introduction of the relative version of (1.1).

Definition 2.1 ([16]) Let $K, E \in \mathcal{K}_{n}^{n}$. The isoperimetric quotient of $K$ relative to $E$ is defined as

$$
\mathrm{I}(K ; E)=\frac{\mathrm{S}(K ; E)^{n}}{\operatorname{vol}(K)^{n-1}} .
$$

The function

$$
\mathrm{I}(\lambda)=\mathrm{I}\left(K_{\lambda} ; E\right),
$$

$\lambda \in(-\mathrm{r}(K ; E), \infty)$, is called the isoperimetric quotient function of $K$ with respect to $E$.

Note that $\mathrm{I}(K ; E)$ is invariant under homotheties of $K$, since the $i$-th relative quermassintegral is positively homogeneous of degree $n-i$, i.e. $\mathrm{W}_{i}(\mu K ; E)=$ $\mu^{n-i} \mathrm{~W}_{i}(K ; E)$ for any $\mu \geq 0$. 
Our goal is to analyse the behaviour of (2.5), focusing on the question whether it is a monotonic function in (some parts of) its domain.

A first naive approach to this matter is the natural question whether there are convex bodies $K, E \in \mathcal{K}_{n}^{n}$ for which the isoperimetric quotient function is constant in $(-\mathrm{r}(K ; E), 0]$. We observe that $K_{-\mathrm{r}(K ; E)}$ has no interior points, thus we need to exclude it.

Tangential bodies provide us with a positive answer to this question.

Definition 2.2 ([26, p. 149, text preceding Lemma 3.1.14]) Let $K, E \in \mathcal{K}_{n}^{n}$ be such that $E \subseteq K$. Then the body $K$ is a tangential body of $E$ if and only if through each boundary point of $K$ there exists a supporting hyperplane of $K$ that also supports $E$.

Indeed, inner parallel bodies and tangential bodies happen to be intrinsically connected by means of a homothety relation. The following result enlightens the close connection between inner parallel bodies and tangential bodies, providing us with a constant isoperimetric quotient function on the range $(-\mathrm{r}(K ; E), 0)$ of inner parallel bodies.

Theorem 2.3 ([26, Lemma 3.1.14]) Let $K, E \in \mathcal{K}_{n}^{n}$ be convex bodies, and let $\lambda \in$ $(-\mathrm{r}(K ; E), 0)$. Then $K_{\lambda}$ is homothetic to $K$ if and only if $K$ is homothetic to a tangential body of $E$.

We notice that if $K$ is a tangential body of $E$, then $\mathrm{r}(K ; E)=1$. It follows that the isoperimetric quotient function $\mathrm{I}(\lambda)$ is constant for $-\mathrm{r}(K ; E)=-1<\lambda \leq 0$ if $K$ is a tangential body of $E$.

Although the relative quermassintegral $\mathrm{W}_{i}(\cdot ; E): \mathcal{K}^{n} \rightarrow \mathbb{R}, i=0, \ldots, n-1$, is, in general, not linear under Minkowski addition and scalar multiplication, the $(n-i)$-th root of $\mathrm{W}_{i}(\cdot ; E)$ is concave. In particular, the $n$-th root of the volume is a concave function. This is a consequence of the fundamental Aleksandrov-Fenchel inequality [26, Theorem 7.3.1].

Theorem 2.4 ([26, Chapter 7])(General Brunn-Minkowski theorem for quermassintegrals). Let $K, L, E \in \mathcal{K}_{n}^{n}, 0 \leq i \leq n-1$, and let $\mu \in[0,1]$. Then,

$$
\mathrm{W}_{i}(\mu K+(1-\mu) L ; E)^{\frac{1}{n-i}} \geq \mu \mathrm{W}_{i}(K ; E)^{\frac{1}{n-i}}+(1-\mu) \mathrm{W}_{i}(L ; E)^{\frac{1}{n-i}} .
$$

For $i=0$, i.e. for the volume, equality holds if and only if $K$ and $L$ are homothetic. If $E$ is smooth and $0 \leq i \leq n-2$, equality holds if and only if $K$ and $L$ are homothetic.

Here, a convex body is said to be smooth if it has only one supporting hyperplane at every point of its boundary. The inequality in Theorem 2.4 can be found in [26, Theorem 7.4.5], the characterization of equality for $i=0$ is given in [26, Theorem 7.1.1], and the equality case for smooth $E$ is a consequence of [26, Theorems 7.4.6 and 7.6.9].

To prove the monotonicity of the quotient function $\mathrm{I}(\lambda)$, the derivative of the volume $\operatorname{vol}\left(K_{\lambda}\right)$, as a function of $\lambda \in(-\mathrm{r}(K ; E), \infty)$, turns out to be crucial. In order to deal with derivatives of the quermassintegrals $\mathrm{W}_{i}\left(K_{\lambda} ; E\right)$, in particular of the volume $\operatorname{vol}\left(K_{\lambda}\right)=W_{0}\left(K_{\lambda} ; E\right)$, we need the following results. 
Lemma 2.5 ([26, Lemma 3.1.13]) For all $K, E \in \mathcal{K}_{n}^{n}, \lambda_{0}, \lambda_{1} \in[-\mathrm{r}(K ; E), \infty)$ and $\mu \in[0,1]$,

$$
\mu K_{\lambda_{0}}+(1-\mu) K_{\lambda_{1}} \subseteq K_{\mu \lambda_{0}+(1-\mu) \lambda_{1}},
$$

i.e. the family $\left(K_{\lambda}\right)_{\lambda \geq-\mathrm{r}(K ; E)}$ of all parallel bodies is concave.

Given fixed $K, E \in \mathcal{K}_{n}^{n}$, we are interested in differentiability properties of the functions

$$
\mathrm{W}_{i}(\lambda)=\mathrm{W}_{i}\left(K_{\lambda} ; E\right)
$$

$\lambda \in(-\mathrm{r}(K ; E), \infty)$, for $i=0, \ldots, n-1$. In the sequel, we write $\frac{\mathrm{d}^{-}}{\mathrm{d} \lambda} f(\lambda), \frac{\mathrm{d}^{+}}{\mathrm{d} \lambda} f(\lambda)$, and $\frac{\mathrm{d}}{\mathrm{d} \lambda} f(\lambda)$ for the left, right, and both-sided derivative of a function $f$, respectively, implicitly stating that this quantity exists.

Theorem 2.4 and Lemma 2.5 yield

$$
\frac{\mathrm{d}^{-}}{\mathrm{d} \lambda} \mathrm{W}_{i}(\lambda) \geq \frac{\mathrm{d}^{+}}{\mathrm{d} \lambda} \mathrm{W}_{i}(\lambda) \geq(n-i) \mathrm{W}_{i+1}(\lambda)
$$

for all $K, E \in \mathcal{K}_{n}^{n}, i=0, \ldots, n-1$ and $\lambda \in(-\mathrm{r}(K ; E), \infty)$ (see [12, formula (1.5)], [26, p. 439, Note 7]).

For $i=0$, i.e. for the case of the volume, even more is known.

Proposition 2.6 Let $K \in \mathcal{K}^{n}, E \in \mathcal{K}_{n}^{n}$. Then, for $-\mathrm{r}(K ; E) \leq \lambda<\infty$, the function $\operatorname{vol}(\lambda)=\operatorname{vol}\left(K_{\lambda}\right)$ is differentiable and satisfies

$$
\frac{\mathrm{d}}{\mathrm{d} \lambda} \operatorname{vol}(\lambda)=n \mathrm{~W}_{1}(\lambda)
$$

with only right derivative for $\lambda=-\mathrm{r}(K ; E)$.

For $\lambda \geq 0$, equation (2.8) (with right derivative at $\lambda=0$ ) is a consequence of (2.2). For $-\mathrm{r}(K ; E) \leq \lambda \leq 0$ (with left derivative at $\lambda=0$ ), we refer to [2,21].

For the particular case $E=B_{n}$, Hadwiger [11, p. 207, formula (30)] has shown that $\frac{\mathrm{d}}{\mathrm{d} \lambda} \operatorname{vol}(\lambda)=\mathrm{S}\left(K_{\lambda}\right)$ for all $\lambda \in\left(-\mathrm{r}\left(K ; B_{n}\right), \infty\right)$. Since $n \mathrm{~W}_{1}(K ; E)=\mathrm{S}(K ; E)$, (2.8) amounts to

$$
\frac{\mathrm{d}}{\mathrm{d} \lambda} \operatorname{vol}(\lambda)=\mathrm{S}\left(K_{\lambda} ; E\right)
$$

Remark 2.7 For $\lambda \geq 0$, the differentiability of all quermassintegrals at $\lambda$ (with right derivative for $\lambda=0)$ follows from (2.3), along with the equality $\frac{\mathrm{d}}{\mathrm{d} \lambda} \mathrm{W}_{i}(\lambda)=(n-$ i) $\mathrm{W}_{i+1}(\lambda)$.

The question for which convex bodies there is equality in (2.7) on $-\mathrm{r}(K ; E)<$ $\lambda<\infty$, in particular at $\lambda=0$, is not completely understood yet. We refer the reader to [12] for some results in this direction. 


\section{Monotonicity of the Isoperimetric Quotient for Parallel Bodies}

In this section, we will prove that the isoperimetric quotient function is decreasing. Our result is prepared by some auxiliary statements. The first one is an immediate consequence of formula (2.9) or Proposition 2.6.

Lemma 3.1 Let $K, E \in \mathcal{K}_{n}^{n}$, and $\lambda \in(-\mathrm{r}(K ; E), \infty)$. Then

$$
\frac{\mathrm{d}}{\mathrm{d} \lambda}\left(\operatorname{vol}(\lambda)^{\frac{1}{n}}\right)=\frac{1}{n} \operatorname{vol}(\lambda)^{\frac{1-n}{n}} \mathrm{~S}\left(K_{\lambda} ; E\right)
$$

The following statement corresponds to formula (18) of [10, Section 6] if $E=B_{n}$. Although the proof is analogous to the proof in the case $E=B_{n}$, we include it for the sake of completeness.

Lemma 3.2 For all $K, E \in \mathcal{K}_{n}^{n}$, and $-\mathrm{r}(K ; E)<\lambda_{0}<\lambda_{1}<\infty$,

$$
K_{\lambda_{0}}=K_{\lambda_{1}} \sim\left|\lambda_{0}-\lambda_{1}\right| E
$$

Proof Let $K, E \in \mathcal{K}_{n}^{n}$, and $-\mathrm{r}(K ; E)<\lambda_{0}<\lambda_{1}$.

Equation (2.1) implies directly $K_{\lambda_{1}} \sim\left|\lambda_{0}-\lambda_{1}\right| E \subseteq K_{\lambda_{0}}$.

For the converse, let $x \in K_{\lambda_{0}}$. Then, by (2.1), $x \in \bigcap_{u \in \mathbb{S}^{n-1}} H_{u, h_{K}(u)+\lambda_{0} h_{E}(u)}^{-}$. Thus,

$x+\left|\lambda_{0}-\lambda_{1}\right| E=x+\left(\lambda_{1}-\lambda_{0}\right) E \subseteq \bigcap_{u \in \mathbb{S}^{n-1}} H_{u,\left(h_{K}(u)+\lambda_{0} h_{E}(u)\right)+\left(\lambda_{1}-\lambda_{0}\right) h_{E}(u)}^{-}=K_{\lambda_{1}}$,

whence $x \in K_{\lambda_{1}} \sim\left|\lambda_{0}-\lambda_{1}\right| E$.

Lemma 3.3 Let $K, L \in \mathcal{K}_{n}^{n}$. If $K+L$ is homothetic to $L$ then $K$ is homothetic to $L$.

Proof Let $K, L \in \mathcal{K}_{n}^{n}$, and let $K+L$ be homothetic to $L$. Then, there are $\alpha>1$ and $x_{0} \in \mathbb{R}^{n}$ such that $K+L=\alpha L+x_{0}$. Hence, rewriting the latter in terms of support functions

$$
h(K, \cdot)+h(L, \cdot)=h(K+L, \cdot)=h\left(\alpha L+x_{0}, \cdot\right)=\alpha h(L, \cdot)+\left\langle x_{0}, \cdot\right\rangle,
$$

this yields

$$
h(K, \cdot)=(\alpha-1) h(L, \cdot)+\left\langle x_{0}, \cdot\right\rangle=h\left((\alpha-1) L+x_{0}, \cdot\right)
$$

and in turn $K=(\alpha-1) L+x_{0}$.

Now, we can prove the monotonicity of the isoperimetric quotient of the family $\left(K_{\lambda}\right)_{\lambda>-\mathrm{r}(K ; E)}$.

Theorem 3.4 Let $K, E \in \mathcal{K}_{n}^{n}$ be convex bodies. Then the function

$$
\mathrm{I}(\lambda)=\frac{\mathrm{S}\left(K_{\lambda} ; E\right)^{n}}{\operatorname{vol}\left(K_{\lambda}\right)^{n-1}}
$$


is monotonically decreasing on $(-\mathrm{r}(K ; E), \infty)$.

Moreover, the following are equivalent for all $-\mathrm{r}(K ; E)<\lambda_{0}<\lambda_{1}<\infty$ :

(i) $\mathrm{I}\left(\lambda_{0}\right)=\mathrm{I}\left(\lambda_{1}\right)$,

(ii) $K_{\lambda_{0}}$ is homothetic to $K_{\lambda_{1}}$,

(iii) $K_{\lambda_{1}}$ is homothetic to a tangential body of $E$,

(iv) $\mathrm{I}(\lambda)$ is constant on $\left(-\mathrm{r}(K ; E), \lambda_{1}\right]$.

If $\lambda_{1}>0$, the equivalent conditions (i)-(iv) are satisfied if and only if $K$ is homothetic to $E$ and, consequently, if and only if $\mathrm{I}(\lambda)=n^{n} \operatorname{vol}(E)$ for all $\lambda \in(-\operatorname{r}(K ; E), \infty)$.

Proof Let $K, E \in \mathcal{K}_{n}^{n}$. Then, the case $i=0$ in Theorem 2.4 and Lemma 2.5 ensure that $\lambda \mapsto \operatorname{vol}(\lambda)^{\frac{1}{n}}$ defines a concave function on $(-\mathrm{r}(K ; E), \infty)$. Further, by Lemma 3.1, the derivative $\frac{\mathrm{d}}{\mathrm{d} \lambda}\left(\operatorname{vol}(\lambda)^{\frac{1}{n}}\right)$ of that function exists, and is monotonically decreasing (see e.g. [24]). Hence, by (3.1), $\mathrm{I}(\lambda)=\left(n \frac{\mathrm{d}}{\mathrm{d} \lambda}\left(\operatorname{vol}(\lambda)^{\frac{1}{n}}\right)\right)^{n}$ decreases as well.

(i) $\Rightarrow$ (ii). Condition (i), together with the just proven monotonicity of the function $\mathrm{I}(\lambda)$, implies that $\mathrm{I}(\lambda)=\left(n \frac{\mathrm{d}}{\mathrm{d} \lambda}\left(\operatorname{vol}(\lambda)^{\frac{1}{n}}\right)\right)^{n}$ is constant on $\left[\lambda_{0}, \lambda_{1}\right]$. Thus, the function $\lambda \mapsto \operatorname{vol}(\lambda)^{\frac{1}{n}}$ is affine on $\left[\lambda_{0}, \lambda_{1}\right]$, and we have equality in (2.6) for $i=0$ and all $\mu \in[0,1]$. Now, by Theorem $2.4, K_{\lambda_{0}}$ and $K_{\lambda_{1}}$ are homothetic.

(ii) $\Rightarrow$ (iii). Let $K_{\lambda_{0}}$ and $K_{\lambda_{1}}$ be homothetic. Then, by Lemma 3.2, we can ensure that the inner parallel body $K_{\lambda_{0}}=K_{\lambda_{1}} \sim\left|\lambda_{1}-\lambda_{0}\right| E$ of $K_{\lambda_{1}}$ is homothetic to $K_{\lambda_{1}}$. Now Theorem 2.3 shows that $K_{\lambda_{1}}$ is homothetic to a tangential body of $E$.

(iii) $\Rightarrow$ (iv). Let $\lambda \in\left(-\mathrm{r}(K ; E), \lambda_{1}\right)$, and $K_{\lambda_{1}}$ be homothetic to a tangential body of $E$. Then, by Lemma 3.2, $K_{\lambda}=K_{\lambda_{1}} \sim\left|\lambda-\lambda_{1}\right| E$ is an inner parallel body of $K_{\lambda_{1}}$. Thus, Theorem 2.3 together with condition (iii), i.e. $K_{\lambda_{1}}$ is homothetic to a tangential body of $E$, implies that $K_{\lambda}$ is homothetic to $K_{\lambda_{1}}$. Since the isoperimetric quotient $K \mapsto \mathrm{I}(K ; E)$ agrees for homothetic bodies, $\mathrm{I}(\lambda)=\mathrm{I}\left(\lambda_{1}\right)$.

(iv) $\Rightarrow$ (i) is trivial.

Now, we deal with the last claim. For that, let $\lambda_{1}>0$, and assume that any of the equivalent assertions (i)-(iv) holds. By (iv), $\mathrm{I}(0)=\mathrm{I}\left(\lambda_{1}\right)$, since $-\mathrm{r}(K ; E)<0<\lambda_{1}$. Then, by (ii), $K_{0}=K$ is homothetic to $K_{\lambda_{1}}=K+\lambda_{1} E$, which together with Lemma 3.3 yields that $K$ is homothetic to $\lambda_{1} E$ and thus $K$ is homothetic to $E$ itself.

Since the isoperimetric quotient $K \mapsto I(K ; E)$ is invariant under homotheties, w.l.o.g. we can assume that $K=E$. Then, $K_{\lambda}=(1+\lambda) E$ for $-\mathrm{r}(K ; E)=-1<\lambda$. Again, by the mentioned invariance, we obtain $\mathrm{I}(\lambda)=\mathrm{I}(E ; E)$ for all $-\mathrm{r}(K ; E)<\lambda$. Finally, $\mathrm{I}(E ; E)=n^{n} \operatorname{vol}(E)$, using that $\mathrm{W}_{i}(E ; E)=\operatorname{vol}(E)$ for all $0 \leq i \leq n$, together with (2.4).

As a corollary, we obtain that the classical isoperimetric quotient is monotonically decreasing, by setting $E=B_{n}$.

Corollary 3.5 For every $K \in \mathcal{K}_{n}^{n}$ and $E=B_{n}$, the function

$$
\mathrm{I}(\lambda)=\frac{\mathrm{S}\left(K_{\lambda}\right)^{n}}{\operatorname{vol}\left(K_{\lambda}\right)^{n-1}}
$$

is monotonically decreasing on $\left(-\mathrm{r}\left(K ; B_{n}\right), \infty\right)$. 
Remark 3.6 It is crucial for the monotonicity in Theorem 3.4 that the surface area is considered relative to the gauge body $E$ : For example, consider $K=[0,1]^{2}$ and $E=[0,1] \times[0,2]$ in $\mathbb{R}^{2}$. Then $K_{0}=K, K_{1}=[0,2] \times[0,3]$, and the classical isoperimetric quotients, where the surface area is taken relative to $B_{2}$, are $\mathrm{I}\left(K_{0}\right)=$ $\frac{4^{2}}{1}=16<\frac{50}{3}=\frac{10^{2}}{6}=\mathrm{I}\left(K_{1}\right)$.

\section{Monotonicity for Related Families of Bodies and Isoperimetric Problems}

In this section, we introduce some new (one-parameter) families of bodies related to the family $\left(K_{\lambda}\right)_{\lambda \geq-\mathrm{r}(K ; E)}$ of parallel bodies. First we recall that a body $K$ is determined by a set $\Omega \subseteq \mathbb{S}^{n-1}$ if

$$
K=\bigcap_{u \in \Omega} H_{u, h_{K}(u)}^{-}
$$

[26, pp. 385, 411].

Remark 4.1 ([26, p. 386]) The smallest closed set $\Omega \subseteq \mathbb{S}^{n-1}$ that determines a given convex body $K$ is the closure of the set $\mathcal{U}(K)$ of outer unit normal vectors at regular boundary points of $K$. In other words, $\Omega \subseteq \mathbb{S}^{n-1}$ determines $K$ if and only if $\mathcal{U}(K) \subseteq$ $\operatorname{cl}(\Omega)$, where $\operatorname{cl}(\cdot)$ denotes the closure operator.

The elements of $\mathcal{U}(K)$ are also known in the literature as extreme normal vectors of $K$. The set $\mathcal{U}(K)$ need not be closed; e.g. when $K \in \mathcal{K}_{2}^{2}$ is a semicircle.

First we prove that in the definition (2.1) of inner parallel bodies of $K \in \mathcal{K}_{n}^{n}$ relative to $E \in \mathcal{K}_{n}^{n}$, we can replace the complete sphere $\mathbb{S}^{n-1}$ in the intersection by any subset $\Omega$ that determines $K$.

Lemma 4.2 Let $K, E \in \mathcal{K}_{n}^{n}$, and let $\Omega \subseteq \mathbb{S}^{n-1}$ determine $K$. Then

$$
K_{\lambda}=\bigcap_{u \in \Omega} H_{u, h_{K}(u)+\lambda h_{E}(u)}^{-}
$$

for all $\lambda \in[-\mathrm{r}(K ; E), 0]$.

Proof Let $K, E \in \mathcal{K}_{n}^{n}$, and let $\Omega \subseteq \mathbb{S}^{n-1}$ determine $K$.

By (2.1), it is evident that $K_{\lambda} \subseteq \bigcap_{u \in \Omega} H_{u, h_{K}(u)+\lambda h_{E}(u)}^{-}$.

For the verification of the reverse inclusion, let $x \in \bigcap_{u \in \Omega} H_{u, h_{K}(u)+\lambda h_{E}(u)}^{-}$be arbitrary. Then, $x \in H_{u, h_{K}(u)-|\lambda| h_{E}(u)}^{-}$for all $u \in \Omega$, i.e. $\langle x, u\rangle \leq h_{K}(u)-|\lambda| h_{E}(u)$ for all $u \in \Omega$. We observe that the latter yields that $x+|\lambda| E \subseteq H_{u, h_{K}(u)}^{-}$for every $u \in \Omega$, since clearly, for any $e \in E$ and $u \in \Omega,\langle x+|\lambda| e, u\rangle \leq h_{K}(u)$.

Consequently,

$$
x+|\lambda| E \subseteq \bigcap_{u \in \Omega} H_{u, h_{K}(u)}^{-}=K,
$$


where the last identity follows from the fact that $\Omega$ determines $K$. Hence, $x \in K_{\lambda}$.

Remark 4.3 Given a set $\Omega \subseteq \mathbb{S}^{n-1}$ that contains the origin in the interior of its convex hull, we define the set

$$
E^{\Omega}=\bigcap_{u \in \Omega} H_{u, h_{E}(u)}^{-} .
$$

Observing that $E \subseteq E^{\Omega}$ and that $h_{E}(u)=h_{E^{\Omega}}(u)$ for $u \in \Omega$, it is clear that $E^{\Omega}$ is a tangential body of $E$. Indeed, it follows from the definition that $E^{\Omega}$ is the smallest tangential body of $E$ that is determined by $\Omega$.

As a particular case of the latter we can obtain the so-called form body of $K$ relative to $E$, for any $K \in \mathcal{K}_{n}^{n}$. This is defined (cf. [26, p. 386]) as

$$
K^{*}=E^{\mathcal{U}(K)} .
$$

We notice that $\mathcal{U}\left(K^{*}\right) \subseteq \operatorname{cl}(\mathcal{U}(K))$ and that the inclusion may be strict [25, Lemma 4.6 and the preceding example].

For fixed $K, E \in \mathcal{K}_{n}^{n}$, and $\Omega \subseteq \mathbb{S}^{n-1}$ determining $K$, Lemma 4.2 motivates the introduction of the following one-parameter family of convex bodies associated to $K$,

$$
K(\Omega, \lambda)=\bigcap_{u \in \Omega} H_{u, h_{K}(u)+\lambda h_{E}(u)}^{-}, \quad \lambda \in[-\mathrm{r}(K ; E), \infty)
$$

Here, the representation (4.1) is naturally extended to $\lambda>0$. The sets $K(\Omega, \lambda)$, $\lambda \in[-\mathrm{r}(K ; E), \infty)$, are, according to [26, Section 7.5], Wulff shapes or Alexandrov bodies associated with the pairs $\left(\Omega, f_{\lambda}\right)$ where $f_{\lambda}: \Omega \rightarrow \mathbb{R}, u \mapsto h_{K}(u)+\lambda h_{E}(u)$.

Our aim is to prove that the relative isoperimetric quotient functions defined for these new one-parameter families of bodies are also decreasing. Before turning to that, we prove the following lemma, which will be useful for later considerations.

Lemma 4.4 Let $K, E \in \mathcal{K}_{n}^{n}$, and let $\Omega \subseteq \mathbb{S}^{n-1}$ determine $K$, i.e. $\mathcal{U}(K) \subseteq \operatorname{cl}(\Omega)$. Then,

(i) $K_{\lambda}=K\left(\mathbb{S}^{n-1}, \lambda\right)$ for all $\lambda \in[-\mathrm{r}(K ; E), \infty)$,

(ii) $K_{\lambda}=K \sim|\lambda| E=K \sim|\lambda| E^{\Omega}=K \sim|\lambda| K^{*}$ for all $\lambda \in[-\mathrm{r}(K ; E), 0]$,

(iii) $K_{\lambda}=K(\Omega, \lambda)$ for all $\lambda \in[-\mathrm{r}(K ; E), 0]$,

(iv) $K+\lambda E=K_{\lambda} \subseteq K(\Omega, \lambda)$ for all $\lambda \geq 0$, and the inclusion may be strict.

Proof Let $K, E \in \mathcal{K}_{n}^{n}$, and let $\Omega \subseteq \mathbb{S}^{n-1}$ so that $\mathcal{U}(K) \subseteq \operatorname{cl}(\Omega)$.

(i) Follows directly from (2.1).

(ii) The first equality is the definition of $K_{\lambda}$. The second one follows from Lemma 4.2 and the observation that $h_{E}(u)=h_{E^{\Omega}}(u)$ for all $u \in \Omega$. Putting $\Omega=\mathcal{U}(K)$ as a particular case, we obtain the last equality.

(iii) Lemma 4.2 
(iv) Follows from (i). To prove that the inclusion may be strict, it is enough to consider in the plane $E=B_{2}, K=[0,1]^{2}$ a square, and $\Omega=\mathcal{U}(K)=\{( \pm 1,0),(0, \pm 1)\}$. Then for any $\lambda>0$, the outer parallel body $K+\lambda B_{2}$ is strictly contained in the square $K(\Omega, \lambda)$.

Next, we state the result about the relative isoperimetric quotient for the family $K(\Omega, \lambda)$ which we aim to prove.

Theorem 4.5 Let $K, E \in \mathcal{K}_{n}^{n}$, and let $\Omega \subseteq \mathbb{S}^{n-1}$ determine $K$. Then the relative isoperimetric quotient function

$$
\mathrm{I}^{\Omega}(\lambda)=\frac{\mathrm{S}(K(\Omega, \lambda) ; E)^{n}}{\operatorname{vol}(K(\Omega, \lambda))^{n-1}}
$$

of the family $(K(\Omega, \lambda))_{\lambda>-\mathrm{r}(K ; E)}$ is monotonically decreasing on $(-\mathrm{r}(K ; E), \infty)$.

Moreover, the following are equivalent for all $-\mathrm{r}(K ; E)<\lambda_{0}<\lambda_{1}<\infty$ :

(i) $\mathrm{I}^{\Omega}\left(\lambda_{0}\right)=\mathrm{I}^{\Omega}\left(\lambda_{1}\right)$,

(ii) $K\left(\Omega, \lambda_{0}\right)$ is homothetic to $K\left(\Omega, \lambda_{1}\right)$,

(iii) $K\left(\Omega, \lambda_{1}\right)$ is homothetic to a tangential body of $E$, and

(iv) $\mathrm{I}^{\Omega}(\lambda)$ is constant on $\left(-\mathrm{r}(K ; E), \lambda_{1}\right]$.

If $\lambda_{1}>0$, the equivalent conditions ( $i$ )-(iv) are satisfied if and only if $K$ is homothetic to $E^{\Omega}$ and, consequently, if and only if $\mathrm{I}(\lambda)=\mathrm{I}\left(E^{\Omega} ; E\right)=n^{n} \operatorname{vol}\left(E^{\Omega}\right)$ for all $\lambda \in(-\mathrm{r}(K ; E), \infty)$.

The proof of Theorem 4.5 is based on the following lemmas.

Lemma 4.6 Let $K, E \in \mathcal{K}_{n}^{n}$, and let $\Omega \subseteq \mathbb{S}^{n-1}$ determine $K$. If $\Lambda>-\mathrm{r}(K ; E)$, then

$$
K(\Omega, \lambda)=(K(\Omega, \Lambda))_{\lambda-\Lambda}, \text { for all } \lambda \in(-\mathrm{r}(K ; E), \Lambda]
$$

Proof Let $K, E \in \mathcal{K}_{n}^{n}$, and let $\Omega \subseteq \mathbb{S}^{n-1}$ determine $K$.

From the definition of $K(\Omega, \Lambda)$ it follows that $K(\Omega, \Lambda)$ is determined by $\Omega$. It follows also from the definition that $h_{K(\Omega, \Lambda)}(u) \leq h_{K}(u)+\Lambda h_{E}(u)$ for all $u \in \Omega$. Using the latter, together with Lemma 4.2, we obtain

$$
\begin{aligned}
(K(\Omega, \Lambda))_{\lambda-\Lambda}= & \bigcap_{u \in \Omega} H_{u, h_{K(\Omega, \Lambda)}(u)+(\lambda-\Lambda) h_{E}(u)}^{-} \\
& \subseteq \bigcap_{u \in \Omega} H_{u,\left(h_{K}(u)+\Lambda h_{E}(u)\right)+(\lambda-\Lambda) h_{E}(u)}^{-}=K(\Omega, \lambda) .
\end{aligned}
$$

To prove the reverse inclusion, let $x \in K(\Omega, \lambda)$. Then $x \in H_{u, h_{K}(u)+\lambda h_{E}(u)}^{-}$for every $u \in \Omega$. As in the proof of Lemma 4.2, it follows that

$$
x+|\lambda-\Lambda| E \subseteq H_{u,\left(h_{K}(u)+\lambda h_{E}(u)\right)+|\lambda-\Lambda| h_{E}(u)}^{-}=H_{u, h_{K}(u)+\Lambda h_{E}(u)}^{-}
$$


for every $u \in \Omega$. Since the last inclusion holds for all $u \in \Omega$, we get $x+|\lambda-\Lambda| E \subseteq$ $K(\Omega, \Lambda)$. That is, $x \in(K(\Omega, \lambda))_{\lambda-\Lambda}$.

Lemma 4.7 Let $K, E \in \mathcal{K}_{n}^{n}$, and let $\Omega \subseteq \mathbb{S}^{n-1}$ determine $K$. Then, for any $\lambda>0$,

$$
h_{K(\Omega, \lambda)}(u)=h_{K}(u)+\lambda h_{E}(u) \quad \text { for all } u \in \Omega \text {. }
$$

Proof Let $K, E \in \mathcal{K}_{n}^{n}$, and let $\Omega \subseteq \mathbb{S}^{n-1}$ determine $K$. Directly from the definition (4.2) of $K(\Omega, \lambda)$, it follows that $h_{K(\Omega, \lambda)}(u) \leq h_{K}(u)+\lambda h_{E}(u)$ for all $u \in \Omega$.

On the other hand, we have

$$
K(\Omega, \lambda)=\bigcap_{u \in \Omega} H_{u, h_{K}(u)+\lambda h_{E}(u)}^{-} \bigcap_{u \in \mathbb{S}^{n-1}} H_{u, h_{K}(u)+\lambda h_{E}(u)}^{-}=K+\lambda E .
$$

From the latter it follows that $h_{K(\Omega, \lambda)}(u) \geq h_{K}(u)+\lambda h_{E}(u)$ for any $u \in \mathbb{S}^{n-1}$. Thus,

$$
h_{K(\Omega, \lambda)}(u)=h_{K}(u)+\lambda h_{E}(u) \quad \text { for all } u \in \Omega \text {. }
$$

Lemma 4.8 Let $K, E \in \mathcal{K}_{n}^{n}$, let $\Omega \subseteq \mathbb{S}^{n-1}$ determine $K$, and let $\lambda>0$. If $K(\Omega, \lambda)$ is homothetic to $K$ then $K$ is homothetic to $E^{\Omega}$.

Proof Let $K, E \in \mathcal{K}_{n}^{n}$ and let $\Omega \subseteq \mathbb{S}^{n-1}$ determine $K$. Let $\lambda>0$ and assume that $K(\Omega, \lambda)$ is homothetic to $K$. Then, there are $\alpha>1$ and $x_{0} \in \mathbb{R}^{n}$ such that $K(\Omega, \lambda)=\alpha K+x_{0}$. Hence, $h_{K(\Omega, \lambda)}=\alpha h_{K}+\left\langle x_{0}, \cdot\right\rangle$. Thus, (4.3) yields

$$
h_{E}(u)=\frac{1}{\lambda}\left((\alpha-1) h_{K}(u)+\left\langle x_{0}, u\right\rangle\right), \quad u \in \Omega .
$$

Finally, it is enough to observe that

$$
\begin{aligned}
E^{\Omega} & =\bigcap_{u \in \Omega} H_{u, h_{E}(u)}^{-}=\bigcap_{u \in \Omega} H_{u, \frac{1}{\lambda}\left((\alpha-1) h_{K}(u)+\left\langle x_{0}, u\right\rangle\right)}^{-} \\
& =\frac{1}{\lambda}\left(\left((\alpha-1) \bigcap_{u \in \Omega} H_{u, h_{K}(u)}^{-}\right)+x_{0}\right)=\frac{1}{\lambda}\left((\alpha-1) K+x_{0}\right) .
\end{aligned}
$$

That is, $K$ is homothetic to $E^{\Omega}$.

Now, we can prove Theorem 4.5

Proof of Theorem 4.5 Let $K, E \in \mathcal{K}_{n}^{n}$ and let $\Omega \subseteq \mathbb{S}^{n-1}$ determine $K$. In order to prove that the relative isoperimetric quotient function $\mathrm{I}^{\Omega}(\lambda)=\frac{\mathrm{S}(K(\Omega, \lambda) ; E)^{n}}{\operatorname{vol}(K(\Omega, \lambda))^{n-1}}$ is decreasing, we will interpret the assertion in terms of the relative (to $E$ ) isoperimetric function $\mathrm{I}(\lambda)=\mathrm{I}\left(L_{\lambda} ; E\right)=\frac{\mathrm{S}\left(L_{\lambda} ; E\right)^{n}}{\operatorname{vol}\left(L_{\lambda}\right)^{n-1}}$ for an appropriate convex body $L$, since for this function we have already proven in Theorem 3.4, that it is decreasing. 
Let $\Lambda>-\mathrm{r}(K ; E)$ be fixed. By Lemma $4.6, K(\Omega, \lambda)=(K(\Omega, \Lambda))_{\lambda-\Lambda}$ for any $\lambda \in(-\mathrm{r}(K ; E), \Lambda]$ and thus, $\mathrm{I}^{\Omega}(\lambda)=\mathrm{I}\left(K(\Omega, \Lambda)_{\lambda-\Lambda} ; E\right)$ for all $\lambda \in$ $(-\mathrm{r}(K ; E), \Lambda]$. Hence, a direct application of Theorem 3.4 to the convex body $K(\Omega, \Lambda)$ gives the claim of Theorem 4.5 for all $\lambda, \lambda_{0}, \lambda_{1} \in(-\operatorname{r}(K ; E), \Lambda]$.

Since this can be done for all $\Lambda>-\mathrm{r}(K ; E)$, the proof of the monotonicity and the equivalence of (i)-(iv) is complete. It only remains to prove the claim concerning $\lambda_{1}>0$.

Let $\lambda_{1}>0$, and assume that any of the equivalent assertions (i)-(iv) holds. Using (iv) we have $\mathrm{I}^{\Omega}(0)=\mathrm{I}^{\Omega}\left(\lambda_{1}\right)$, since $-\mathrm{r}(K ; E)<0<\lambda_{1}$. Now, (ii) shows that $K(\Omega, 0)=K$ is homothetic to $K\left(\Omega, \lambda_{1}\right)$, which together with Lemma 4.8 yields that $K$ is homothetic to $E^{\Omega}$.

Finally, if $K$ is homothetic to $E^{\Omega}$, the invariance under homotheties of the isoperimetric quotient provides us with the last assertion. Indeed, assume that $K=E^{\Omega}=\bigcap_{u \in \Omega} H_{u, h_{E}(u)}^{-}$w.l.o.g., then $K(\Omega, \lambda)=(1+\lambda) E^{\Omega}$ and $\mathrm{I}^{\Omega}(\lambda)=$ $\mathrm{I}^{\Omega}\left((1+\lambda) E^{\Omega} ; E\right)=\mathrm{I}\left(E^{\Omega} ; E\right)$ for $-\mathrm{r}(K ; E)=-1<\lambda$. Finally, since $E^{\Omega}$ is a tangential body of $E$, [26, Theorem 7.6.17] gives $\mathrm{W}_{0}\left(E^{\Omega} ; E\right)=\mathrm{W}_{1}\left(E^{\Omega} ; E\right)$; i.e. $\operatorname{vol}\left(E^{\Omega}\right)=\frac{1}{n} \mathrm{~S}\left(E^{\Omega} ; E\right)$. Thus, $\mathrm{I}\left(E^{\Omega} ; E\right)=n^{n} \operatorname{vol}\left(E^{\Omega}\right)$, which finishes the proof.

Remark 4.9 We have obtained Theorem 4.5 as a consequence of Theorem 3.4. Conversely, Theorem 3.4 follows from Theorem 4.5 , because $K_{\lambda}=K\left(\mathbb{S}^{n-1}, \lambda\right)$ by Lemma 4.4(i), and in turn $\mathrm{I}(\lambda)=\mathrm{I}^{\mathbb{S}^{n-1}}(\lambda)$.

Let us have a look at relations between the classes $\left(K_{\lambda}\right)_{\lambda \geq-\mathrm{r}(K ; E)}$ and $(K(\Omega, \lambda))_{\lambda \geq-\mathrm{r}(K ; E)}$.

Proposition 4.10 Let $K, E \in \mathcal{K}_{n}^{n}$, let $\Omega_{1}, \Omega_{2} \subseteq \mathbb{S}^{n-1}$ both determine $K$, and suppose that $\Omega_{1} \subseteq \Omega_{2}$. Then

$$
K\left(\Omega_{1}, \lambda\right) \supseteq K\left(\Omega_{2}, \lambda\right) \supseteq K_{\lambda}
$$

for all $\lambda \geq-\mathrm{r}(K ; E)$. Furthermore,

(i) for $\lambda \leq 0$, there is equality all over (4.4);

(ii) if $\lambda>0$, then both $K\left(\Omega_{1}, \lambda\right)$ and $K\left(\Omega_{2}, \lambda\right)$ are tangential bodies of $K_{\lambda}$, and moreover, $K\left(\Omega_{1}, \lambda\right)$ is a tangential body of $K\left(\Omega_{2}, \lambda\right)$.

Proof Let $K, E \in \mathcal{K}_{n}^{n}$, and let $\Omega_{1}, \Omega_{2} \subseteq \mathbb{S}^{n-1}$ both determine $K$.

The relations (4.2) and (2.1) directly yield (4.4).

(i) follows from Lemma 4.2.

(ii): Let $\lambda \geq 0$. Then Lemma 4.7 shows that $h_{K\left(\Omega_{1}, \lambda\right)}(u)=h_{K_{\lambda}}(u)$ for all $u \in \Omega_{1}$. Consequently, $K\left(\Omega_{1}, \lambda\right)$ is a tangential body of $K_{\lambda}$, since $K\left(\Omega_{1}, \lambda\right)$ is determined by $\Omega_{1}$ according to its definition (4.2). By the same arguments, $K\left(\Omega_{2}, \lambda\right)$ is a tangential body of $K_{\lambda}$.

Finally, since $K\left(\Omega_{1}, \lambda\right)$ is a tangential body of $K_{\lambda}$, inclusions (4.4) imply that $K\left(\Omega_{1}, \lambda\right)$ is also a tangential body of $K\left(\Omega_{2}, \lambda\right)$, because every common supporting hyperplane of $K\left(\Omega_{1}, \lambda\right)$ and $K_{\lambda}$ supports $K\left(\Omega_{2}, \lambda\right)$, too. 
Next, we consider the asymptotic behaviour of $K_{\lambda}$ and $K(\Omega, \lambda)$ for $\lambda \rightarrow \infty$. We obtain in particular that the inclusions in (4.4) may be strict for large $\lambda$, since the respective limit shapes can be different.

Proposition 4.11 Let $K, E \in \mathcal{K}_{n}^{n}$, and let $\Omega \subseteq \mathbb{S}^{n-1}$ determine $K$. Then the convex bodies $K_{\lambda}$ and $K(\Omega, \lambda)$ converge in $\lambda$ in the sense that

$$
\lim _{\lambda \rightarrow \infty} \frac{1}{\lambda} K_{\lambda}=E \quad \text { and } \quad \lim _{\lambda \rightarrow \infty} \frac{1}{\lambda} K(\lambda, \Omega)=E^{\Omega}
$$

in the Hausdorff metric. Moreover,

$\lim _{\lambda \rightarrow \infty} \mathrm{I}(\lambda)=\mathrm{I}(E ; E)=n^{n} \operatorname{vol}(E) \quad$ and $\lim _{\lambda \rightarrow \infty} \mathrm{I}^{\Omega}(\lambda)=\mathrm{I}\left(E^{\Omega} ; E\right)=n^{n} \operatorname{vol}\left(E^{\Omega}\right)$.

We remark that the convergence result and the limit value for $K_{\lambda}$ can, in its essence, be found in $[25$, p. 56].

Proof The convergences of the bodies follow from their definitions, i.e. from (2.1) and (4.2). The remaining claims $\mathrm{I}(E ; E)=n^{n} \operatorname{vol}(E)$ and $\mathrm{I}\left(E^{\Omega} ; E\right)=n^{n} \operatorname{vol}\left(E^{\Omega}\right)$ have been shown in the proofs of Theorems 3.4 and 4.5 , respectively.

Remark 4.12 Suppose that $\Omega$ determines both $K$ and $E$. Then the families $\left(K_{\lambda}\right)_{\lambda \geq-\mathrm{r}(K ; E)}$ and $(K(\Omega, \lambda))_{\lambda \geq-\mathrm{r}(K ; E)}$ agree for $\lambda \leq 0$ and have the same limit shape $E=E^{\Omega}$. Nevertheless, they do not necessarily coincide. In fact, [25, pp. 23-24] and [16, Section 3] give examples of polytopes $K, E \in \mathcal{K}_{3}^{3}$ such that $\mathcal{U}(K)=\mathcal{U}(E) \subsetneq \mathcal{U}(K+E)$. Then $K_{1} \neq K(\mathcal{U}(K), 1)$, because $K(\mathcal{U}(K), 1)$ is determined by $\mathcal{U}(K)$, whereas $K_{1}=K+E$ is not.

Next, we pose a natural question in this context, which we have not been able to answer so far.

Question 4.13 Let $K, E \in \mathcal{K}_{n}^{n}$, let $\Omega_{1}, \Omega_{2} \subseteq \mathbb{S}^{n-1}$ both determine $K$ and suppose that $\Omega_{1} \subseteq \Omega_{2}$. We know that $\mathrm{I}^{\Omega_{1}}(\lambda)=\mathrm{I}^{\Omega_{2}}(\lambda)$ for $-\mathrm{r}(K ; E)<\lambda \leq 0$ and that $\lim _{\lambda \rightarrow \infty} \mathrm{I}^{\Omega_{1}}(\lambda) \geq \lim _{\lambda \rightarrow \infty} \mathrm{I}^{\Omega_{2}}(\lambda)$. Do we have $\mathrm{I}^{\Omega_{1}}(\lambda) \geq \mathrm{I}^{\Omega_{2}}(\lambda)$ also for all $\lambda>0$ ?

We come to isoperimetrically optimal bodies.

Corollary 4.14 Let $E \in \mathcal{K}_{n}^{n}$ and let $\Omega \subseteq \mathbb{S}^{n-1}$ be a set that contains the origin in the interior of its convex hull.

Then a convex body $\tilde{K} \in \mathcal{K}_{n}^{n}$ is a minimizer of the relative isoperimetric quotient $\mathrm{I}(K ; E)$ among all convex bodies $K \in \mathcal{K}_{n}^{n}$ that are determined by $\Omega$ if and only if $\tilde{K}$ is homothetic to the tangential body $E^{\Omega}$ of $E$. In particular, that minimal quotient is $\mathrm{I}\left(E^{\Omega} ; E\right)=n^{n} \operatorname{vol}\left(E^{\Omega}\right)$.

Proof Let $K \in \mathcal{K}_{n}^{n}$ be determined by $\Omega$. By Theorem 4.5 and Proposition 4.11,

$$
\mathrm{I}(K ; E)=\mathrm{I}^{\Omega}(0) \geq \lim _{\lambda \rightarrow \infty} \mathrm{I}^{\Omega}(\lambda)=\mathrm{I}\left(E^{\Omega} ; E\right)=n^{n} \operatorname{vol}\left(E^{\Omega}\right)
$$


with equality if and only if $\mathrm{I}^{\Omega}(\lambda)$ is constant for $\lambda \geq 0$. The latter holds if and only if all convex bodies $K(\Omega, \lambda), \lambda \geq 0$, are mutually homothetic, and in turn are homothetic to $\lim _{\lambda \rightarrow \infty} \frac{1}{\lambda} K(\Omega, \lambda)=E^{\bar{\Omega}}$.

Remark 4.15 If $E=B_{n}$, Corollary 4.14 concerns the classical isoperimetric quotient from (1.1). For that case Corollary 4.14 is a well-known result [26, p. 385], that goes back to Lindelöf and Minkowski [19,22] for finite $\Omega$ and to Aleksandrov [1] for general $\Omega$.

For $E=B_{n}$ and $\Omega=\mathbb{S}^{n-1}$, we obtain the isoperimetric inequality for arbitrary convex bodies.

\section{Isoperimetric-Type Quotients of Quermassintegrals}

This section is motivated by the following monotonicity result from [16].

Proposition 5.1 ([16, Proposition 4.3, Remark 4.4]) Let $K, E \in \mathcal{K}_{n}^{n}$, let $0 \leq i<j<$ $n$, and suppose that $K$ belongs to the class $\mathcal{R}_{j}$. Then the function

$$
\lambda \mapsto \frac{\mathrm{W}_{j}\left(K_{\lambda} ; E\right)^{n-i}}{\mathrm{~W}_{i}\left(K_{\lambda} ; E\right)^{n-j}}
$$

is monotonically decreasing on $(-\mathrm{r}(K ; E), \infty)$.

Here, the classes $\mathcal{R}_{j}$ are defined by a differentiability condition of the functions $\lambda \mapsto \mathrm{W}_{i}\left(K_{\lambda} ; E\right)$ on $[-\mathrm{r}(K ; E), \infty)$.

Definition 5.2 ([12]) Let $E \in \mathcal{K}_{n}^{n}$ and let $p$ be an integer, $0 \leq p \leq n-1$. A convex body $K \in \mathcal{K}^{n}$ belongs to the class $\mathcal{R}_{p}$ if, for all $0 \leq i \leq p$ and for $-\mathrm{r}(K ; E) \leq \lambda<\infty$, the following equalities hold

$$
\frac{\mathrm{d}^{-}}{\mathrm{d} \lambda} \mathrm{W}_{i}(\lambda)=\frac{\mathrm{d}^{+}}{\mathrm{d} \lambda} \mathrm{W}_{i}(\lambda)=(n-i) \mathrm{W}_{i+1}(\lambda),
$$

where the first equation is to be dropped when $\lambda=-\mathrm{r}(K ; E)$.

We remark that the above definition is natural taking (2.7) into account. Proposition 2.6 yields

$$
\mathcal{R}_{0}=\mathcal{K}^{n}
$$

for any $E \in \mathcal{K}_{n}^{n}$. Clearly,

$$
\mathcal{R}_{n-1} \subseteq \mathcal{R}_{n-2} \subseteq \ldots \subseteq \mathcal{R}_{1} \subseteq \mathcal{R}_{0}
$$

and the inclusions are strict in general, as is shown in [12].

The case $i=0, j=1$ in Proposition 5.1 gives the monotonicity of $\mathrm{I}(\lambda)$ proven in Theorem 3.4, but only under the additional assumption $K \in \mathcal{R}_{1}$. In a similar way as 
we have relaxed the assumptions (to none) in the case $i=0, j=1$ in Theorem 3.4, we shall see next that, more generally, the assumption $K \in \mathcal{R}_{j}$ from Proposition 5.1 can be relaxed by $K \in \mathcal{R}_{j-1}$. We need the following lemma.

Lemma 5.3 Let $0 \leq i \leq n-1, E \in \mathcal{K}_{n}^{n}, K \in \mathcal{R}_{i}$, and $-\mathrm{r}(K ; E)<\lambda<\infty$. Then

$$
\frac{\mathrm{d}}{\mathrm{d} \lambda}\left(\mathrm{W}_{i}(\lambda)^{\frac{1}{n-i}}\right)=\mathrm{W}_{i}(\lambda)^{\frac{1-n+i}{n-i}} \mathrm{~W}_{i+1}(\lambda)
$$

Proof The result follows immediately from the definition of the class $\mathcal{R}_{i}$ and the standard rules of differentiation.

Proposition 5.4 Let $0 \leq i \leq n-2, K, E \in \mathcal{K}_{n}^{n}$, and $K \in \mathcal{R}_{i}$. Then the function

$$
\mathrm{I}_{i}(\lambda)=\frac{\mathrm{W}_{i+1}\left(K_{\lambda} ; E\right)^{n-i}}{\mathrm{~W}_{i}\left(K_{\lambda} ; E\right)^{n-i-1}}
$$

is monotonically decreasing on $(-\mathrm{r}(K ; E), \infty)$.

Moreover, if $E$ is smooth, the following are equivalent for all $-\mathrm{r}(K ; E)<\lambda_{0}<$ $\lambda_{1}<\infty$ :

(i) $\mathrm{I}_{i}\left(\lambda_{0}\right)=\mathrm{I}_{i}\left(\lambda_{1}\right)$,

(ii) $K_{\lambda_{0}}$ is homothetic to $K_{\lambda_{1}}$,

(iii) $K_{\lambda_{1}}$ is homothetic to an $(n-i-1)$-tangential body of $E$, and

(iv) $\mathrm{I}_{i}(\lambda)$ is constant on $\left(-\mathrm{r}(K ; E), \lambda_{1}\right]$.

If $E$ is smooth and $\lambda_{1}>0$, conditions $(i)$-( $\left.i v\right)$ are satisfied if and only if $K$ is homothetic to $E$ and, consequently, if and only if $\mathrm{I}_{i}(\lambda)=\operatorname{vol}(E)$ for all $\lambda \in(-\mathrm{r}(K ; E), \infty)$.

The case $i=n-1$ is excluded, since $\mathrm{I}_{n-1}(\lambda)=\mathrm{W}_{n}\left(K_{\lambda} ; E\right)=\operatorname{vol}(E)$ is constant.

Before dealing with the proof, we introduce a refined definition of tangential bodies (of a fixed convex body), for which we need the notion of $p$-extreme supporting hyperplanes, $0 \leq p \leq n-1$. Given a convex body $K \in \mathcal{K}_{n}^{n}$, a supporting hyperplane $H_{u, h_{K}(u)}$ of $K$ is called $(n-p-1)$-extreme [26, p. 85] if its outer normal vector $u \in \mathbb{R}^{n} \backslash\{0\}$ cannot be represented as a sum of $n-p+1$ linearly independent outer normal vectors at the same boundary point of $K$.

Definition 5.5 ([26, p. 86]) Let $K, E \in \mathcal{K}_{n}^{n}$ be such that $E \subseteq K$, and let $p \in$ $\{0, \ldots, n-1\}$. Then $K$ is a $p$-tangential body of $E$ if each $(n-p-1)$-extreme supporting hyperplane of $K$ is a supporting hyperplane of $E$.

We observe that $K$ is a tangential body of $E$ in the sense of Definition 2.2 if and only if $K$ is just an $(n-1)$-tangential body of $E$ [26, pp. 86, 149].

Remark 5.6 Let $E \in \mathcal{K}_{n}^{n}$.

(i) Every $p$-tangential body of $E$ is also a $q$-tangential body of $E$ whenever $0 \leq$ $p<q \leq n-1$.

(ii) The only 0-tangential body of $E$ is $E$ itself. 
(iii) A tangential body $K$ of $E$ belongs to the class $\mathcal{R}_{p}$ if and only if $K$ is an $(n-p-1)$ tangential body of $E$ [12, Theorem 1.3].

Proof of Proposition 5.4 Let $0 \leq i \leq n-2$, and let $K \in \mathcal{K}_{n}^{n}$ lie in the class $\mathcal{R}_{i}$. By Lemma 5.3 and the assumption of $K \in \mathcal{R}_{i}$, the derivative $\frac{\mathrm{d}}{\mathrm{d} \lambda}\left(\mathrm{W}_{i}(\lambda)^{\frac{1}{n-i}}\right)$ exists and satisfies (5.2). Theorem 2.4 and Lemma 2.5 ensure that $\lambda \mapsto \mathrm{W}_{i}(\lambda)^{\frac{1}{n-i}}$ defines a concave and differentiable function on $(-\mathrm{r}(K ; E), \infty)$. Thus, the derivative $\frac{\mathrm{d}}{\mathrm{d} \lambda}\left(\mathrm{W}_{i}(\lambda)^{\frac{1}{n-i}}\right)$ is monotonically decreasing (see e.g. [24]). Hence, by (5.2), $\mathrm{I}_{i}(\lambda)=\left(\frac{\mathrm{d}}{\mathrm{d} \lambda}\left(\mathrm{W}_{i}(\lambda)^{\frac{1}{n-i}}\right)\right)^{n-i}$ decreases, too.

We notice that the statements (i)-(iv) are the exact analogues of the statements (i)(iv) in Theorem 3.4, with the only addendum of $K$ being an $(n-i-1)$-tangential body of $E$ instead of just a tangential body of $E$. However, these two assertions are equivalent for bodies $K \in \mathcal{R}_{i}$ by Remark 5.6. Hence, the proof of the equivalences of (i)-(iv) can follow the respective steps of the proof of Theorem 3.4.

For the last part of the proof, we can proceed in analogy to the proof of Theorem 3.4, too. Indeed, note that from the homogeneity of quermassintegrals, once obtained $K_{\lambda}=$ $(1+\lambda) E$, it follows that $\mathrm{I}_{i}(\lambda)=\operatorname{vol}(E)$ by using that $\mathrm{W}_{j}(E ; E)=\operatorname{vol}(E)$ for any $0 \leq j \leq n$.

We observe that for $i=0$, we recover Theorem 3.4 (notice the multiplicative constant $n$ from (2.4)), since the class $\mathcal{R}_{0}$ consists of all convex bodies.

Now, we obtain the announced improvement of [16, Proposition 4.3] (i.e. of Proposition 5.1) as a corollary.

Theorem 5.7 Let $K, E \in \mathcal{K}_{n}^{n}$, let $0 \leq i<j<n$, and suppose that $K$ belongs to the class $\mathcal{R}_{j-1}$. Then the function

$$
\mathrm{I}_{i, j}(\lambda)=\frac{\mathrm{W}_{j}\left(K_{\lambda} ; E\right)^{n-i}}{\mathrm{~W}_{i}\left(K_{\lambda} ; E\right)^{n-j}}
$$

is monotonically decreasing on $(-\mathrm{r}(K ; E), \infty)$.

Moreover, if $E$ is smooth, the following are equivalent for all $-\mathrm{r}(K ; E)<\lambda_{0}<$ $\lambda_{1}<\infty$ :

(i) $\mathrm{I}_{i, j}\left(\lambda_{0}\right)=\mathrm{I}_{i, j}\left(\lambda_{1}\right)$,

(ii) $K_{\lambda_{0}}$ is homothetic to $K_{\lambda_{1}}$,

(iii) $K_{\lambda_{1}}$ is homothetic to an $(n-j)$-tangential body of $E$, and

(iv) $\mathrm{I}_{i, j}(\lambda)$ is constant on $\left(-\mathrm{r}(K ; E), \lambda_{1}\right]$.

If $E$ is smooth and $\lambda_{1}>0$, conditions (i)-(iv) are satisfied if and only if $K$ is homothetic to $E$ and, consequently, if and only if $\mathrm{I}_{i, j}(\lambda)=\operatorname{vol}(E)^{j-i}$ for all $\lambda \in$ $(-\mathrm{r}(K ; E), \infty)$.

Proof Let $K, E \in \mathcal{K}_{n}^{n}$, let $0 \leq i<j<n$, and let $K$ belong to the class $\mathcal{R}_{j-1}$. Since $K \in \mathcal{R}_{j-1}$, we know from (5.1), that $K \in \mathcal{R}_{k}$ for $k=i, \ldots, j-1$. Proposition 5.4 shows that all the functions $\mathrm{I}_{k}(\lambda), k=i, \ldots, j-1$, decrease. Then 


$$
\prod_{k=i}^{j-1} \mathrm{I}_{k}(\lambda)^{\frac{(n-i)(n-j)}{(n-k-1)(n-k)}}=\frac{\mathrm{W}_{j}\left(K_{\lambda} ; E\right)^{n-i}}{\mathrm{~W}_{i}\left(K_{\lambda} ; E\right)^{n-j}}=\mathrm{I}_{i, j}(\lambda)
$$

decreases as well.

Moreover, we obtain $\mathrm{I}_{i, j}\left(\lambda_{0}\right)=\mathrm{I}_{i, j}\left(\lambda_{1}\right)$ for some $-\mathrm{r}(K ; E)<\lambda_{0}<\lambda_{1}$ if and only if $\mathrm{I}_{k}\left(\lambda_{0}\right)=\mathrm{I}_{k}\left(\lambda_{1}\right)$ for $k=i, \ldots, j-1$. The characterizations of the last assertions, given in Proposition 5.4, yield the remainder of Proposition 5.7.

The following result is now obtained in the same way as Corollary 4.14 was proven using Theorem 4.5.

Corollary 5.8 Let $0 \leq i<j<n$ and let $E \in \mathcal{K}_{n}^{n}$ be smooth. Then a convex body $\tilde{K} \in \mathcal{R}_{j-1} \cap \mathcal{K}_{n}^{n}$ is a minimizer of the quotient $\frac{\mathrm{W}_{j}(K ; E)^{n-i}}{\mathrm{~W}_{i}(K ; E)^{n-j}}$ among all convex bodies $K \in \mathcal{R}_{j-1} \cap \mathcal{K}_{n}^{n}$ if and only if $\tilde{K}$ is homothetic to $E$.

The assumption $K \in \mathcal{R}_{j-1}$ in Theorem 5.7 and Corollary 5.8, respectively, is essential in our proof. However, so far we do not have an example of a body $K \in$ $\mathcal{K}_{n}^{n} \backslash \mathcal{R}_{j-1}$ that does not satisfy the claims of Proposition 5.7 or Corollary 5.8.

Remark 5.9 Theorem 3.4 on the family $\left(K_{\lambda}\right)_{\lambda>-\mathrm{r}(K ; E)}$ of parallel bodies gave rise to the analogous Theorem 4.5 on the families $(K(\Omega, \lambda))_{\lambda>-\mathrm{r}(K ; E)}$, since the last families could be interpreted as inner parallel bodies by Lemma 4.6. In a similar way, results of the present section imply analogues concerning the families $(K(\Omega, \lambda))_{\lambda>-\mathrm{r}(K ; E)}$. Then conditions $K \in \mathcal{R}_{i}$ have to be replaced by $K(\Omega, \Lambda) \in \mathcal{R}_{i}$ for all $\Lambda>-\mathrm{r}(K ; E)$.

Funding Open Access funding enabled and organized by Projekt DEAL.

Open Access This article is licensed under a Creative Commons Attribution 4.0 International License, which permits use, sharing, adaptation, distribution and reproduction in any medium or format, as long as you give appropriate credit to the original author(s) and the source, provide a link to the Creative Commons licence, and indicate if changes were made. The images or other third party material in this article are included in the article's Creative Commons licence, unless indicated otherwise in a credit line to the material. If material is not included in the article's Creative Commons licence and your intended use is not permitted by statutory regulation or exceeds the permitted use, you will need to obtain permission directly from the copyright holder. To view a copy of this licence, visit http://creativecommons.org/licenses/by/4.0/.

\section{References}

1. Alexandrov, A.D.: Zur Theorie der gemischten Volumina von konvexen Körpern, III: Die Erweiterung zweier Lehrsätze Minkowskis über die konvexen Polyeder auf beliebige konvexe Flächen (in Russian). Mat. Sbornik N. S. 3, 27-46 (1938)

2. Bol, G.: Beweis einer Vermutung von H. Minkowski. Abh. Math. Sem. Univ. Hamburg 15, 37-56 (1943)

3. Bonnesen, T., Fenchel, W.: Theorie der konvexen Körper. Springer, Berlin, 1934, 1974. English translation: Theory of convex bodies. Edited by L. Boron, C. Christenson and B. Smith. BCS Associates, Moscow, ID (1987)

4. Crasta, G.: Anisotropic perimeter and isoperimetric quotient of inner parallel bodies, arXiv:2101.03307

5. Dinghas, A.: Bemerkung zu einer Verschärfung der isoperimetrischen Ungleichung durch H. Hadwiger. Math. Nachr. 1, 284-286 (1948) 
6. Dinghas, A.: Über eine neue isoperimetrische Ungleichung für konvexe Polyeder. Math. Ann. 120, 533-538 (1949)

7. Domokos, G., Lángi, Z.: The isoperimetric quotient of a convex body decreases monotonically under the Eikonal abrasion model. Mathematika 65(1), 119-129 (2019)

8. Gardner, R., Kiderlen, M.: A solution to Hammer's X-ray reconstruction problem. Adv. Math. 214(1), 323-343 (2007)

9. Gruber, P.M.: Convex and Discrete Geometry. Springer, Berlin Heidelberg (2007)

10. Hadwiger, H.: Altes und Neues über konvexe Körper. Birkhäuser Verlag, Basel und Stuttgart (1955)

11. Hadwiger, H.: Vorlesungen über Inhalt. Oberfläche und Isoperimetrie. Springer-Verlag, BerlinGöttingen-Heidelberg (1957)

12. Hernández Cifre, M.A., Saorín, E.: On differentiability of quermassintegrals. Forum Math. 22(1), 115-126 (2010)

13. Hernández Cifre, M.A., Saorín, E.: On the volume of inner parallel bodies. Adv. Geom. 10(2), 275-286 (2010)

14. Hernández Cifre, M.A., Saorín, E.: On inner parallel bodies and quermassintegrals. Israel J. Math. 177, 29-47 (2010)

15. Hernández Cifre, M.A., Saorín, E.: Differentiability of quermassintegrals: a classification of convex bodies. Trans. Amer. Math. Soc. 366, 591-609 (2014)

16. Hernández Cifre, M.A., Saorín, E.: Isoperimetric relations for inner parallel bodies, to appear in Commun. Anal. Geom.

17. Henk, M., Wills, J.M.: A Blichfeldt-type inequality for the surface area. Monatsh. Math. 154, 135-144 (2008)

18. Larson, S.: A bound for the perimeter of inner parallel bodies. J. Funct. Anal. 271, 610-619 (2016)

19. Lindelöf, L.: Propriétés générales des polyèdres qui, sous une étendue superficielle donnée, renferment le plus grand volume. Bull. Acad. Sci. St. Pétersbourg 4, 257-269 (1869). Extract: Math. Ann. 2, 150159 (1870)

20. Linke, E., Saorín Gómez, E.: Decomposition of polytopes using inner parallel bodies. Monatsh. Math. 176(4), 575-588 (2015)

21. Matheron, G.: La formule de Steiner pour les érosions. J. Appl. Prob. 15, 126-135 (1978)

22. Minkowski, H.: Allgemeine Lehrsätze über die convexen Polyeder, Nachr. Ges. Wiss. Göttingen, 198219. Gesammelte Abhandlungen, vol. II, pp. 103-121, Teubner, Leipzig (1911)

23. Rolfes, J.H., Vallentin, F.: Covering compact metric spaces greedily. Acta Math. Hung. 155(1), 130-140 (2018)

24. Royden, H.L.: Real Analysis, 3rd edn. Macmillan Publishing Company, New York (1988)

25. Sangwine-Yager, J.R.: Inner Parallel Bodies and Geometric Inequalities. Ph.D. Thesis Dissertation, University of California Davis (1978)

26. Schneider, R.: Convex Bodies: The Brunn-Minkowski Theory. Cambridge University Press, Cambridge Second Expanded Edition (2014)

27. Steiner, J.: Über parallele Flächen. Monatsber. Preuß. Akad. Wiss., Berlin (1840), 114-118. Also: Gesammelte Werke, vol. 2, pp. 171-176, Reimer, Berlin (1882)

Publisher's Note Springer Nature remains neutral with regard to jurisdictional claims in published maps and institutional affiliations. 